\title{
Research on Credit Risk Evaluation of SMEs in Supply Chain Finance based on Big Data
}

\author{
Xiaofei Zhang ${ }^{1, \mathrm{a}}$, Hongmei Zhang ${ }^{2, \mathrm{~b}}$ \\ ${ }^{1,2}$ School of Big Data Application and Economics, Guizhou University of Finance and Economics, Guiyang Guizhou 550025, China \\ a542663288@qq.com, bzhm1035@qq.com

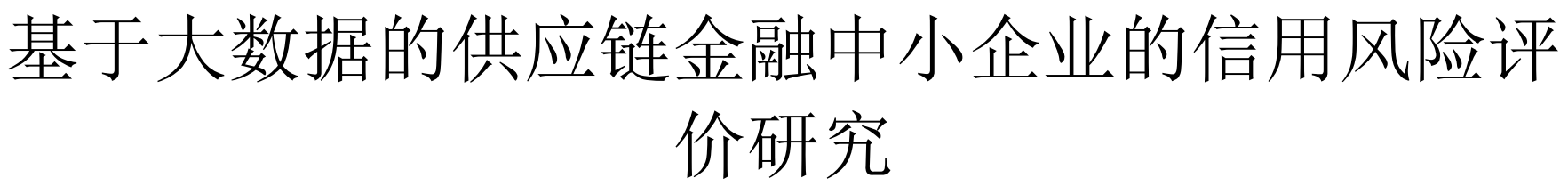 \\ 奕晓飞 $1, \mathrm{a}$, 张红梅 $2, \mathrm{~b}$ \\ 1,2 贵州财经大学大数据应用与经济学院, 花溪, 贵阳, 贵州, 550025, 中国 \\ a542663288@qq.com, bzhm1035@qq.com
}

\begin{abstract}
With the development of global supply chain, enterprises are more and more aware of the advantages of supply chain. In recent years, supply chain finance has become the focus of many logistics enterprises and financial institutions. In this paper, principal component analysis and logistic regression method are used to further analyze the credit risk of SMEs under the supply chain financial model, so as to provide a theoretical basis for improving the credit risk assessment method and making correct decisions.
\end{abstract}

Keywords—supply chain finance, credit risk, logistic model

摘要一随着全球供应链的发展, 企业越来越意识到供应链 的优势。近年来供应链金融已经成为许多物流企业、金融机 构关注的焦点。本文通过采用主成分分析法和 logistic 模型 回归法, 进一步的分析供应链金融模式下中小企业的信用风 险状况，从而为完善信用风险评估方法、做出正确的决策提 出理论依据。

关键词一供应链金融, 信用风险, logistic 模型

\section{I. 引言}

较部分西方国家，我国的供应链金融概念起步较 晚, 约在 20 世纪 80 年代至今, 这近四十年的时间里, 供应链金融一路经历了线下、线下线上相结合、电商供 应链金融及多元供应链金融四个大的阶段。

在我国现有的经济市场大环境下，中小企业融资 难, 融资贵依然是难解决的问题, 而且有八成的企业在 这范畴之内。一方面是个体工商户、企业之间建立在一 定合作、信任基础上的㻌账、㻌销现象明显。或因流动 资金不足，或因需要放大自身资金利用率等其它原因， 使得被㻌个体、企业资金压力增大，被迫需要融资; 另 一方面中小企业资金流十分脆弱。信息的不对称，征信 体系的缺失，造成了银行及其他同梯队金融机构对于其 资质的审核难度达到最大，审核周期长、授信低成为了 中小企业融资的 “两座大山”。种种原因使得中小企业 融资渠道被迫转向嗜血的次级金融机构一民间借贷机 构，融资成本居高不下，十分不利于中小企业良性发 展。而供应链金融模式最大的特点就是以供应链中的核 心企业为出发点，一方面将资金有效注入处于相对弱势
的上下游链属企业; 另一方面将银行信用融入链属企业 的购销行为, 增强其商业信用。

因此, 本文通过 logistic 模型对供应链金融企业的信 用风险进行评价研究, 来确定这些中小企业的信用风 险, 具有现实意义。

\section{II. 文献回顾}

Michael Porter(1985)提出了价值链理论, 从此出现了 供应链一词, 但是 “供应链金融” 是在 2003 年受到学者 们的关注。Y.Sheffi(2003)[1]将供应链与金融结合起来然 后应用于供应链优化决策中, 并且论述了其可能性和它 的潜在价值。Allen N.Berger 和 Gregory F.Udell(2004)[2] 通过总结一些成功的案例, 对中小企业的融资提出了新 的想法, 这初步确定了供应链金融的思想。Hofmsnn.E (2005)[3]第一次对供应链金融内涵进行了界定一它融 合了供应链管理、物流学、金融学这几门学科的共同之 处, 将物流、资金流、信息流进行整合从而实现供应链 上各个节点企业的价值增值。Feinberg(2007)[4]指出供应 链金融是可以由一系列可以为供应链融资中任意阶段提 供资金的融通的融资模式组成。, 因此他认为供应链金融 是其金融供应链的一个组成部分。 Michael Lamoureux(2008)[5]把核心企业作为出发点, 通过成本分 析、成本管理和各种融资手段对供应链中资金的可得性 和成本进行优化。Leora Kapper[6]、Seda Ugurlu 和 Cengiz Kahraman[7]通过实证检验发现供应链金融在经济 发展良好的国家应用的比较广泛, 并因此提升了信用信 息的管理水平, 通过以存货融资模式说明信用良好的交 易企业对融资企业进行担保可以很好地减轻借款者的信 息不透明问题，从而促进双方的共同发展。

在国内, 王蝉 (2006) [8]从整个产业链的视角出 发, 认为银行应转变为针对整条供应链进行风险管理, 借助经济实力较为雄厚的信誉良好的核心企业来为供应 链各节点企业提供全面的独到的资金融通服务, 进而优 化整条供应链。张琅（2010）[9]以供应链视角下的中小 融资企业信用风险综合评估问题为研究中心, 并从供应 链金融概念的界定、供应链金融信用风险影响因素的分 
析、供应链金融信用风险评估指标体系的构建及评估模 型的建立四个方面展开, 通过比较研究找到适合我国商 业银行使用的供应链金融信用风险评估方法，运用主成 分分析法、 logistic 模型, 从而为我国商业银行更加合 理、有效地实施供应链金融信用风险管理提出科学可行 的建议。胡海青等人 (2011) [10]考虑到在供应链金融 中，虽然银行间的信用等级评估标准不同，但最后面临 的都是是否贷款给中小企业这样一个二类分类问题，他 们运用支持向量机（SVM）法建立基于支持向量机的供 应链金融信用风险评估模型。王晓龙（2013）[11]针对 供应链金融风险控制系统的分析和初步设计, 给帮助建 设供应链金融的风险控制信息系统体系提出方案。从供 应链融资模式角度, 针对存货质押融资、应收账款融 资、预付款融资三种典型的融资模式，从功能和数据上 分析应如何风险控制。夏立明等（2013）[12]针对供应 链金融中的市场风险, 将时间维的思想运用到了供应链 金融中小企业信用风险评价模型中，并使用微粒群算法 确定指标权重, 最后通过实证分析来检验该模型的科学 性和有效性。

\section{III. 供应链金融模式及信用风险理论分析}

\section{A. 供应链金融}

供应链，通过对信息流、物流、资金流的控制，从 采购原材料开始, 制成中间产品以及最终产品, 最后由 销售网络把产品送到消费者手中，将供应商、制造商、 分销商、零售商、最终用户连成一个整体的功能网链结 构。它不仅时一条连接供应商到用户的物流链、信息 链、资金链, 而且是一条增值链, 物料在供应链上因加 工、包装、运输等过程而增加其价值，给相关企业带来 收益。

供应链金融是商业银行信贷业务的一个专业领域 （银行层面），也是企业尤其是中小企业的一种融资渠 道（企业层面）。

它是指银行向核心企业提供融资和其他结算、理财 服务，同时向这些核心企业的供应商提供贷款及时收达 的便利，或者向其分销商提供预付款代付及存货融资服 务 (简单地说, 就是银行将核心企业和上下游企业联系 在一起提供灵活运用的金融产品和服务的一种融资模 式）。

\section{B. 供应链金融模式}

供应链金融的实质是帮助企业盘活流动资产，即 应收、预付和存货。因此通常将产品分为三类：应收 类、预付类和存活类。

\section{C. 供应链金融信用风险的界定}

本文认为供应链金融信用风险是指在供应链金融操 作的过程中，供应链上的中小企业由于市场因素、经营 能力、道德水平、管理能力等自身因素或其他因素影响 导致其违反约定事项或无法履约, 使银行实际收益与预 期目标发生背离，从而给银行带来信贷风险的可能性。

\section{D. 供应链金融信用风险评估办法}

logistic 回归(Logistic regression)与多重线性回归实际 上有很多相同之处，最大的区别就在于他们的因变量不
同，其他的基本都差不多，正是因为如此，这两种回归 可以归于同一个家族, 即广义线性模型（generalized linear model）。这一家族中的模型形式基本上都差不 多, 不同的就是因变量不同, 如果是连续的, 就是多重 线性回归, 如果是二项分布, 就是 logistic 回归, 如果是 poisson 分布, 就是 poisson 回归, 如果是负二项分布, 就是负二项回归, 等等。只要注意区分它们的因变量就 可以了。

logistic 回归的因变量可以是二分非线性差分方程类 的, 也可以是多分类的, 但是二分类的更为常用, 也更 加容易解释。所以实际中最为常用的就是二分类的 logistic 回归。

\section{IV. 供应链金融信用风险评价指标体系的构建}

\section{A. 供应链金融模式下指标体系的构建}

我们选取的指标分别能体系企业的盈利能力、营运 能力、偿债能力和发展前景。其中盈利能力中选取营业 利润率、净资产收益率和资产净利率这几个指标。营运 能力选取总资产周转率、存货周转率和应收账款周转 率。偿债能力中选取流动比率、现金流动负债比和资本 负债率。发展前景中选取净利润增长率、营业收入增长 率和总资产增长率。

\section{B. 供应链金融模式下中小企业信用风险评估的模型选 择}

目前, 我国的信用数据库建设时间不长, 现代信用 风险的度量模型对于数据的要求比较高, 从而要使用传 统的信用风险度量模型。而在一些传统的信用风险度量 模型中, 专家制度法的主观性太强, 没有相应的数据理 论作为支撑; 信用评级法主观性也很强, 除此之外由于 我国目前的信用评级公司发展落后、评级标准不统一导 致准确性不强; 神经网络模型可以弱化指标权重的影 响, 但由于工作上的随机性, 使其使用在商业银行对中 小企业的贷款信用评价中有一定的风险。

信用评分法中的 LOGISTIC 回归模型是使用一系列 指标来预测企业的守约概率, 不要求数据服从正态分 布, 与其它方法相比更适合分析供应链金融模式下中小 企业的信用风险评估。

\section{V. 运用 LOGISTIC 模型对中小企业信用风险的评估}

\section{A. 样本数据的选取}

本文主要选取中小企业板块 36 家上市公司作为研究 对象，其中 9 家为违约风险较大、非正常上市公司， 27 家为正常合格的公司。本文初步选择了 12 个定量指标对 供应链模式下中小企业得信用风险状况进行评估。通过 变量之间的相关系数矩阵可知, 变量与变量之间的相关 性较大, 直接用于模型分析会导致较大的多重共线性, 因此在做 logistic 回归分析之前, 先对指标进行预处理, 通过提取主成分因子法最大限度的将解释变量保留在模 型中, 从而达到降维的效果。因此, 本文首先对这 35 个 指标进行分析, 将其分为今个包含原指标大部分信息的 不想关的指标。

\section{1) 数据的检验}


因子分析是以最少的信息损失, 将众多的原始变量浓 缩成少数几个公因子,使得变量具有更高的可解释性的一 种多元统计方法。本文用 SPSS 软件进行因子分析,表 1 给出了因子分析的 KMO 检验和球形 Bartlett 检验结果。 Bartlett 球形检验的概率 $p$ 值为 0.000 ,即原假设被拒绝,也 就是说, 可以认为相关系数矩阵与单位矩阵有显著差别。 同时 $\mathrm{KMO}$ 值为 0.577 ,根据 $\mathrm{KMO}$ 度量可知,原变量适合 进行因子分析。

表 $1 K M O$ 和 Bartlett 的检验

\begin{tabular}{lrr}
\hline \multicolumn{3}{c}{ KMO 和 Bartlett 的检验 } \\
\hline 取样足够度的 Kaiser-Meyer-Olkin 度量 & 0.577 \\
& 近似卡方 & 97.847 \\
Bartlett 的球形度检验 & $d f$ & 36 \\
& Sig. & 0.000 \\
\hline
\end{tabular}

2) 因子的提取

表 2 解释的总方差

\begin{tabular}{|c|c|c|c|c|c|c|c|c|}
\hline \multirow{3}{*}{$\begin{array}{l}\text { 成 } \\
\text { 份 }\end{array}$} & \multicolumn{2}{|c|}{ 初始特征值 } & \multicolumn{3}{|c|}{ 提取平方和载入 } & \multicolumn{3}{|c|}{ 旋转平方和载入 } \\
\hline & 方差 & & & 方差 & & & 方差 & \\
\hline & 的\% & 累积\% & 合计 & 的\% & 累积\% & 合计 & 的\% & 累积 \% \\
\hline
\end{tabular}

$\begin{array}{llllllllll}1 & 4.373 & 29.155 & 29.155 & 4.373 & 29.155 & 29.155 & 3.251 & 21.671 & 21.671\end{array}$

$\begin{array}{llllllllll}2 & 3.489 & 23.257 & 52.412 & 3.489 & 23.257 & 52.412 & 3.124 & 20.830 & 42.501\end{array}$

$\begin{array}{llllllllll}3 & 2.147 & 14.312 & 66.723 & 2.147 & 14.312 & 66.723 & 2.425 & 16.170 & 58.670\end{array}$

$\begin{array}{llllllllll}4 & 1.461 & 9.738 & 76.461 & 1.461 & 9.738 & 76.461 & 2.055 & 13.697 & 72.368\end{array}$

$\begin{array}{llllllllll}5 & 1.294 & 8.628 & 85.089 & 1.294 & 8.628 & 85.089 & 1.908 & 12.721 & 85.089\end{array}$

$\begin{array}{llll}6 & 0.769 & 5.130 & 90.219\end{array}$

在因子分析的过程中，本文选用特征值大于 1 的指 标进行分析, 共提取了 5 个成分, 其累计贡献率达到了 $85.09 \%$ 选取 $F_{1}, F_{2}, F_{3}, F_{4}, F_{5}$ 作为最终指标进行分 析。

\section{3) 因子解释}

表 3 旋转成份矩阵

\begin{tabular}{lccccc}
\hline & \multicolumn{5}{c}{ 成份 } \\
& $\mathbf{1}$ & $\mathbf{2}$ & $\mathbf{3}$ & $\mathbf{4}$ & $\mathbf{5}$ \\
\hline 净资产收益率(TTM)(\%)_ROETTM & 0.943 & 0.207 & 0.061 & 0.080 & 0.004 \\
资产净利率(TTM)(\%)_ROATTM & 0.911 & 0.200 & 0.069 & 0.162 & -0.018 \\
营业利润率(\%)_Opeprfrt & 0.855 & 0.124 & 0.149 & 0.290 & 0.056 \\
净利润增长率(\%)_Netprfgrrt & 0.703 & -0.008 & 0.057 & -0.101 & -0.055 \\
营业收入增长率(\%)_Opeincmgrrt & 0.085 & 0.873 & 0.312 & -0.010 & -0.040 \\
营业收入增长率(\%)_Opeincmgrrt & 0.085 & 0.873 & 0.312 & -0.010 & -0.040 \\
总资产周转率(次)_Totassrat & 0.209 & 0.797 & -0.215 & -0.165 & 0.312 \\
总资产周转率(次)_Totassrat & 0.209 & 0.797 & -0.215 & -0.165 & 0.312 \\
总资产增长率(\%)_Totassgrrt & 0.130 & 0.101 & 0.946 & 0.021 & -0.031 \\
总资产增长率(\%)_Totassgrrt & 0.130 & 0.101 & 0.946 & 0.021 & -0.031 \\
流动比率(\%)_Currt & 0.063 & -0.150 & 0.159 & 0.892 & -0.117 \\
现金流动负债比_OpeCcurdb & 0.051 & 0.043 & -0.368 & 0.751 & -0.025 \\
资产负债率(\%)_Dbastrt & -0.393 & 0.249 & -0.388 & -0.706 & 0.020 \\
应收账款周转率(次)_ARTrat & 0.024 & -0.020 & -0.060 & -0.023 & 0.947 \\
存货周转率(次)_Invtrtrrat & -0.097 & 0.350 & 0.026 & -0.119 & 0.890 \\
\hline
\end{tabular}

通过因子的提取, 可以找到模型的主成分因子。但 是每个变量都可以由多个因子变量所解释, 从而导致了 每一个因子都不能全面的解释原有变量, 使得某一个因 自变量的意义模棱两可。本文通过旋转成分矩阵的方法 来解决这个问题。表 3 是对 5 个因子成分做出经济解 释。

第一因子 $F_{l}$ 主要解释了净资产收益率、资产净利率 和营业利润率, 它们在因子上的载荷分别为 0.943 、 $0.911 、 0.855$, 说明该因子主要反映了企业的盈利能 力, 因此该因子命名为盈利因子。

第二因子 $F_{2}$ 主要解释了营业收入增长率、总资产增 长率、存货周转率, 它们在因子上的载荷分别为 $0.873 、 0.797 、 0.350$, 说明该因子主要反映了企业的发 展前景, 因此该因子命名为成长因子。

第三因子 $F_{3} 、 F_{4}$ 主要解释了流动比率、总资产增长 率、资产负债率、现金流动负债比, 它们在因子上的载 荷分别为 $0.892 、 0.946 、 0.368 、 0.388$, 说明该因子主要 反映了企业的偿债能力, 因此这两个因子总命名为偿债 因子。

第五因子 $F_{5}$ 主要解释了应收账款周转率、存货周转 率、总资产周转率, 它们在因子上的载荷分别为 $0.947 、 0.890 、 0.312$, 说明该因子主要反映了企业的营 运能力, 因此该因子命名为营运因子。

4) 因子得分

表 4 成份得分系数矩阵

\begin{tabular}{lccccc}
\hline & \multicolumn{5}{c}{ 成份 } \\
& $\mathbf{1}$ & $\mathbf{2}$ & $\mathbf{3}$ & $\mathbf{4}$ & $\mathbf{5}$ \\
\hline 净资产收益率(TTM)(\%)_ROETTM & 0.323 & -0.025 & -0.049 & -0.075 & -0.014 \\
资产净利率(TTM)(\%)_ROATTM & 0.298 & -0.011 & -0.048 & -0.025 & -0.021 \\
营业利润率(\%)_Opeprfrt & 0.261 & -0.033 & -0.004 & 0.050 & 0.047 \\
流动比率(\%)_Currt & -0.107 & 0.054 & 0.029 & 0.483 & 0.012 \\
现金流动负债比_OpeCcurdb & -0.079 & 0.131 & -0.204 & 0.453 & -0.008 \\
营业收入增长率(\%)_Opeincmgrrt & -0.095 & 0.339 & 0.087 & 0.079 & -0.124 \\
总资产增长率(\%)_Totassgrrt & -0.028 & -0.016 & 0.408 & -0.036 & 0.041 \\
总资产周转率(次)_Totassrat & 0.023 & 0.249 & -0.121 & 0.000 & 0.049 \\
资产负债率(\%)_Dbastrt & -0.048 & 0.078 & -0.131 & -0.305 & -0.093 \\
营业收入增长率(\%)_Opeincmgrrt & -0.095 & 0.339 & 0.087 & 0.079 & -0.124 \\
净利润增长率(\%)_Netprfgrrt & 0.285 & -0.099 & -0.020 & -0.175 & -0.030 \\
总资产增长率(\%)_Totassgrrt & -0.028 & -0.016 & 0.408 & -0.036 & 0.041 \\
存货周转率(次)_Invtrtrrat & -0.060 & 0.014 & 0.072 & 0.038 & 0.479 \\
应收账款周转率(次)_ARTrat & 0.023 & -0.145 & 0.045 & 0.034 & 0.565 \\
总资产周转率(次)_Totassrat & 0.023 & 0.249 & -0.121 & 0.000 & 0.049 \\
\hline
\end{tabular}

其中:

令净资产收益率为 $X_{1}$, 资产净利率为 $X_{2}$, 营业利润 率为 $X_{3}$, 流动比率为 $X_{4}$, 现金流动负债比为 $X_{5}$, 营业 收入增长率为 $X_{6}$, 总资产增长率为 $X_{7}$, 总资产周转率 为 $X_{8}$, 资产负债率为 $X_{9}$, 营业收入增长率为 $X_{10}$, 净利润 增长率为 $X_{11}$, 总资产增长率为 $X_{12}$,

存货周转率为 $X_{13}$, 应收账款周转率为 $X_{14}$, 总资产 
周转率为 $X_{15}$ 。

\section{则根据表 4 所得:}

$F_{I}=0.323 X_{I}+0.298 X_{2}+0.261 X_{3}-0.107 X_{4}-0.079 X_{5}-$ $0.095 X_{6}-0.028 X_{7}+0.023 X_{8}-0.048 X_{9}-0.095 X_{10}+0.285 X_{11^{-}}$ $0.028 X_{12}-0.060 X_{13}+0.023 X_{14}+0.023 X_{15}$

$$
\text { ...... }
$$$$
F_{5}=-0.014 X_{1}-0.021 X_{2}+0.047 X_{3}+0.012 X_{4}-0.008 X_{5}
$$

$0.124 X_{6}+0.041 X_{7}+0.049 X_{8}-0.093 X_{9}+0.124 X_{10^{-}}$

$0.030 X_{11}+0.041 X_{12}+0.479 X_{13}+0.565 X_{14}+0.049 X_{15}$

根据上述公式可以计算出各因子的得分情况, 其中 $X_{1} \ldots \ldots X_{15}$ 均为标准化之后的数据。

\section{B. logistic 回归分析及违约判别模型的建立}

本文使用 logistic 模型对样本进行回归, 以是否存在 违约情况作为因变量, 将 3 家可能存在违约状况的上市 公司赋值为 1 , 将 9 家合格经营的上市公司赋值为 0 , 将上文分析的 5 个因子作为自变量, 建立二分类的 logistic 模型, 回归结果如表:

表 5 模型系数的综合检验

\begin{tabular}{rlrrr}
\hline \multicolumn{5}{c}{ 模型系数的综合检验 } \\
\hline & & & \\
\hline & & 卡方 & $d f$ & \multicolumn{1}{c}{ Sig. } \\
步骤 1 & 步骤 & 9.208 & 3 & 0.006 \\
& 块 & 16.228 & 9 & 0.000 \\
& 模型 & 16.228 & 9 & 0.000 \\
\hline
\end{tabular}

表 6 模型汇总

\begin{tabular}{cccc}
\hline \multicolumn{3}{c}{ 模型汇总 } \\
\hline 步骤 & -2 对数似然值 & Cox \& Snell R 方 & Nagelkerke R 方 \\
1 & $24.261 \mathrm{a}$ & 0.363 & 0.537 \\
\hline
\end{tabular}

从表 5 模型系数的综合检验中, 其中模型一行输出 了 logistic 回归模型中所有参数是否均为 0 的似然比检 验结果。 $P<0.05$ 表示本次拟合的模型中, 纳入的变量 中, 至少有一个变量的 $O R$ 值有统计学意义, 即模型总 体有意义, 在此表格中, 模型一行的 $s i g$ 为 $0.000<0.005$, 则证明此模型有意义。

模型汇总是检验模型的拟合优度。当 $P$ 值不小于检 验水准时 (即 $P>0.05$ ), 认为当前数据中的信息已经被 充分提取, 模型拟合优度高。从表 6 所得, 此时 $P$ 值为 $0.537>0.05$, 则证明此模型拟合优度高。

\section{表 7 分类表}

\begin{tabular}{|c|c|c|c|c|}
\hline \multicolumn{5}{|c|}{ 分类表 } \\
\hline \multirow{5}{*}{ 步骤 1} & \multirow{2}{*}{\multicolumn{2}{|c|}{ 已观测 }} & $\begin{array}{r}\text { 已预测 } \\
\text { 是否存在违约风险 }\end{array}$ & 百分比 \\
\hline & & & 否 是 & 校正 \\
\hline & 是否存在违 & 0 & 25 & 92.6 \\
\hline & 约风险 & 1 & 3 & 66.7 \\
\hline & 总计百分比 & & & 86.1 \\
\hline
\end{tabular}

通过表 7 可知，在分析的 36 家企业中，不存在违约 风险的 27 家中，模型可以正确分析出 25 家企业（违约 状况 0 代表不存在违约状况), 错误判断出 2 家, 模型 的准确率高达 $92.6 \%$; 在存在违约风险的 9 家企业中,
模型可以正确分析出 6 家, 错误判断出 3 家, 模型的准 确率达到 $66.7 \%$, 综合分析得出模型的判断准确率达到 了 $86.1 \%$ 。

\section{VI. 结论}

供应链金融服务的创新发展, 为突破中小企业融资 困难的瓶颈, 拓宽融资渠道, 加强供应链的整体竞争力 提供了切实可行的解决方案。发展供应链金融业务虽然 能带来 “共赢” 效果。但同样存在各种各样的风险。有 效地分析和控制这些风险是供应链金融能否成功的关键 之一。因此结合风险来源加强相应的风险管理, 有效控 制风险，是供应链金融业务能否成功的关键。

本文从中小企业的角度出发, 在参考总结前人研究 成果的基础上, 从对供应链金融中小企业信用风险评价 研究中, 主要得出的理论和实证研究结论如下:

（1）构建了供应链金融中小企业信用风险评价指标 体系。本文从盈利能力、营运能力、偿债能力以及成长 能力等方面选取指标因子, 从而构建了一套完整的指标 体系。

（2）建立了 logistic 模型, 有利于进行供应链融资 模式下的中小企业信用风险评估, 并且还验证了此模型 的合理性和科学性。

在此论文的基础上我们得知, 要想化解供应链金融 潜在的风险, 应该从多方面人手, 涵盖整个供应链的各 个环节, 包括对核心企业跟踪评价, 成立物流金融公 司, 提高对中小企业的风险识别控制能力。银行贷款制 度创新并规范内部操作环节, 加强信用整合并建立灵活 的市场商品和反馈体系等。通过以上措施, 以期达到减 少和防范风险, 从而实现供应链金融可持续发展的目 的。

\section{致谢}

本研究得到国家自然科学基金地区项目《贷款风险补 偿资金对科技型中小企业信贷配给的影响机理研究》 (71263011)、《基于文本信息的科技型中小企业信用风 险识别机理研究》(71861003)的大力资助。

\section{参考文献}

[1] Y Sheffi, "Supply chain management under the threat of international terrorism," The International Journal of Logistics Management, vol.12, issue2, pp.1-11, 2003.

[2] Alen N Berains,ger, Gregory F Udell, "A more conceptual framework for SME Financial," Work bank conference on Small and Medium Enterprises Overcoming Growth Constraints, vol.7, issue2, MC13121, pp.14-15, October, 2004.

[3] Hofmann E, "Supply chain finance: some conceptual insights," Logistic Management Innovative Logistic concepts, vol.1, issue 2, pp.203-214, 2005

[4] Susan Feinberg, "So You Think You Understand Supply Chain Finance," Logistic Management-Innovative Logistic concept, pp.3648, 2007(7).

[5] Michael Lamoureux, "A Supply Chain Finance Prime," Supply Chain Finance, pp.34-48, 2007(4).

[6] Leora Klapper, "The Role of Reverse Factoring in Supplier Financing of Small and Medium Sized Enterprise," World Bamk, pp.102-103, 2004.

[7] Seda Ugurlu, Cengiz Kahraman, "Fuzzy Multicriteria Risk Assessment for Hazardous Waste Management: The Case of 
Istanbul,” Journal of Risk Analysis and Crisis Response, vol.1, issue 1, pp.29-41, August 2011.

[8] 王蝉, “中小企业融资新途径--金融服务,”财经界(下半月), pp.9798, 2006(1).

[9] 张琅, “供应链金融信用风险评估研究,”西安理工大学, 2010.
[10] 胡海青, 张琅, 张道宏, 陈亮, “基于支持向量机的供应链金融信 用风险评信研究,”软科学, vol2, issue2, pp.26-30, 2011(5).

[11] 王晓龙., “基于供应链金融的风险控制系统的设计, ”复旦大学, 2013.

[12] 夏立明,边亚男,宗恒恒, “基于供应链金融的中小企业信用风险评 价模型研究,”商业研究, pp.171-177, 2013(10) 